Document downloaded from:

http://hdl.handle.net/10251/140879

This paper must be cited as:

Lamprou, E.; Aguilar -Talens, A.; Gonzalez-Montoro, A.; Monzó Ferrer, JM.; CañizaresLedo, G.; Iranzo-Egea, S.; Vidal San Sebastian, LF.... (21-1). PET detector block with accurate 4D capabilities. Nuclear Instruments and Methods in Physics Research Section A Accelerators Spectrometers Detectors and Associated Equipment. 912:132-136. https://doi.org/10.1016/j.nima.2017.11.002

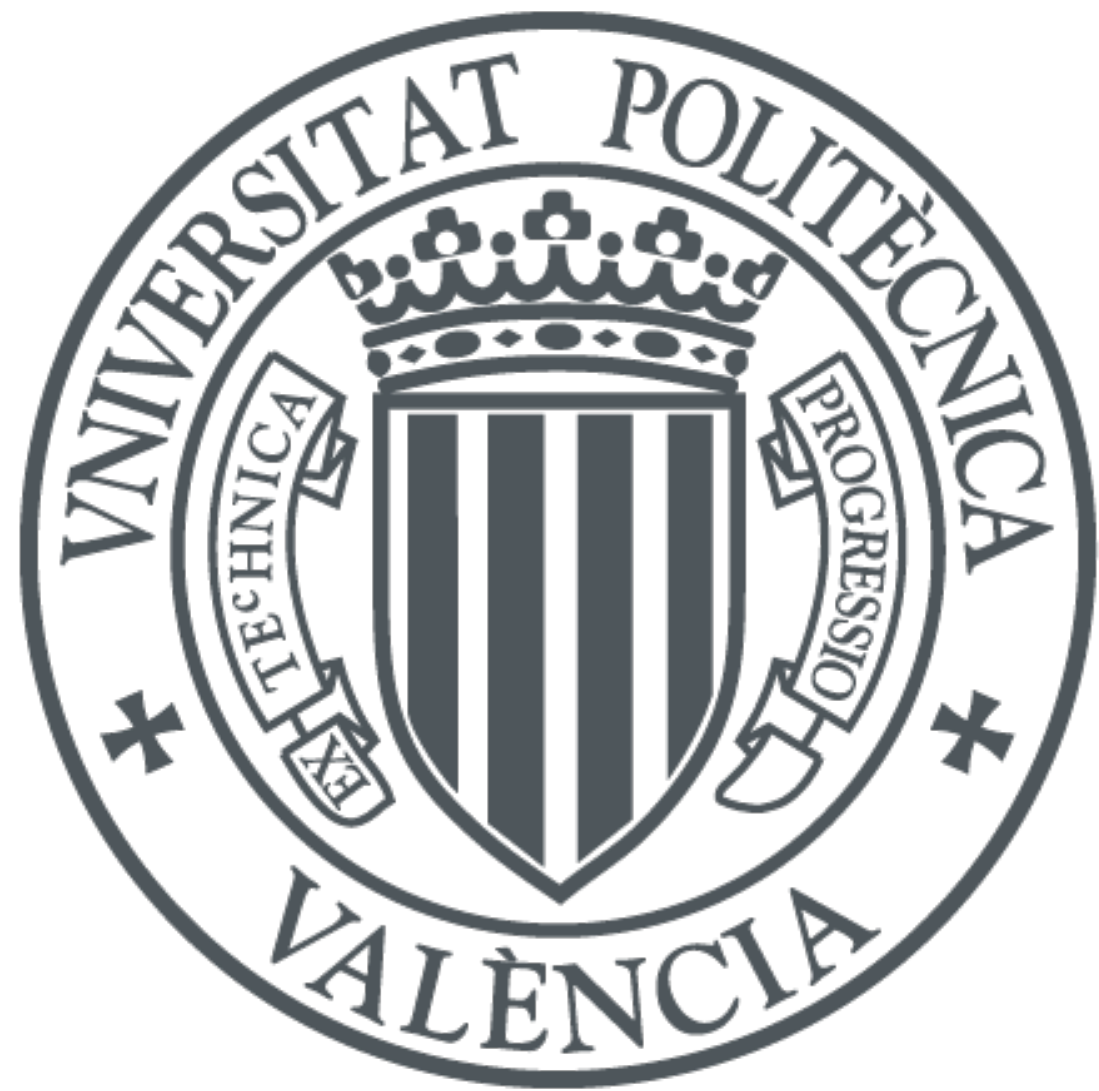

The final publication is available at

https://doi.org/10.1016/j.nima.2017.11.002

Copyright Elsevier

Additional Information 


\title{
PET Detector Block with Accurate 4D Capabilities
}

\author{
Efthymios Lamprou*, Albert Aguilar, Andrea González-Montoro, Jose M. Monzó, \\ Gabriel Cañizares, Sofia Iranzo, Luis F. Vidal, Liczandro Hernández, Pablo Conde, \\ Sebastian Sánchez, Filomeno Sánchez, Antonio J. González and José M. Benlloch \\ Instituto de Instrumentación para Imagen Molecular (I3M), Centro Mixto CSIC - Universitat Politècnica de València, Camino de Vera s/n, \\ 46022 Valencia, SPAIN
}

Elsevier use only: Received date here; revised date here; accepted date here

\begin{abstract}
In this contribution, large SiPM arrays $\left(8 \times 8\right.$ elements of $6 \times 6 \mathrm{~mm}^{2}$ each) are processed with an ASIC-based readout and coupled to a monolithic LYSO crystal to explore their potential use for TOF-PET applications. The aim of this work is to study the integration of this technology in the development of clinical PET systems reaching sub-300 ps coincidence resolving time (CRT). The SiPM and readout electronics have been evaluated first, using a small size $1.6 \mathrm{~mm}(6 \mathrm{~mm}$ height $)$ crystal array $(32 \times 32$ elements). All pixels were well resolved and they exhibited an energy resolution of about $20 \%$ (using Timeover-Threshold methods) for the $511 \mathrm{keV}$ photons. Several parameters have been scanned to achieve the optimum readout system performance, obtaining a CRT as good as $330 \pm 5$ ps FWHM. When using a black-painted monolithic block, the spatial resolution was measured to be on average $2.6 \pm 0.5 \mathrm{~mm}$, without correcting for the source size. Energy resolution appears to be slightly above $20 \%$. CRT measurements with the monolithic crystal detector were also carried out. Prelimnary results as well as calibration methods especifically designed to improve timing performance, are being analyzed in the present manuscript.
\end{abstract}

Keywords: Dedicated PET system; Limited Angle Tomography; Time of flight; TOF-PET; ASIC; SiPM; Monolithic crystals

PACS: the PACS codes can be found at the home page of NIMA (left column, under Contents Services):

http://www1.elsevier.com/homepage/sak/pacs/homepacs.htm

${ }^{*}$ Corresponding author e-mail: e.lamprou@i3m.upv.es 


\section{Introduction}

Whole body PET scanners exhibit some limitations when imaging small organs or lesions. The concept of dedicated PET systems offers a variety of advantages in the clinical practice. In some cases, these systems follow geometries different from fully closed rings. Figure 1 depicts, as an example, a possible implementation for a cardiac PET under stress situation. The missing angular information, when using two panels geometries, may introduce artifacts visible in the reconstructed images across the Field-of-View, worsening the final system performance [1]. However, it has been shown that accurate information on the detected photons timing (Time-ofFlight, TOF) can significantly reduce these effects [2].

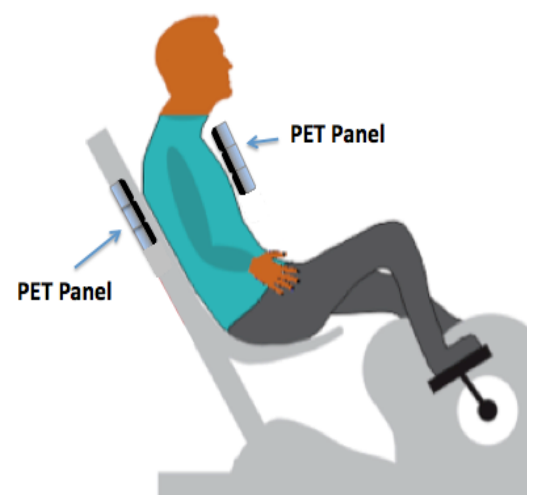

Fig. 1. Sketch of a heart PET with limited angle tomography geometry, where the patient is in a stress situation while cycling.

Our proposal consists of using high resolution PET detectors based on monolithic scintillators capable to provide precise TOF. Monolithic scintillators blocks allow one for the accurate determination of the threedimensional photon impact coordinates, as they typically preserve the scintillation light distribution profile. In addition, we suggest using Application Specific Integrated Circuits (ASIC) readout allowing us to independently read each SiPM pixel and making it possible to achieve optimal TOF performance. All together, 3D spatial and timing resolution, is what we dubbed 4D capabilities.

Building PET detector blocks based on monolithic scintillators crystals infer some challenges. First, a wider scintillation light distribution compared to crystal arrays, results in a high number of SiPM pixels that are hit per 
1 photon impact, affecting the signal-to-noise-ratio (SNR). The 4D impact determination is also affected by the

2 increase in dark counts arising from the photosensors. Moreover, strong influence by the time walk error is being observed as a result of the low SNR. For the current case of light sharing among several SiPMs, the timestamp of the event is deduced from averaging methods among the known individual timestamps [3]. In this work, we present calibration methods designed to overcome these limitations. Experimental results both with monolithic and pixelated scintillator crystals coupled to SiPMs, are presented and evaluated. Promising results are obtained in terms of energy, spatial and timing resolution.
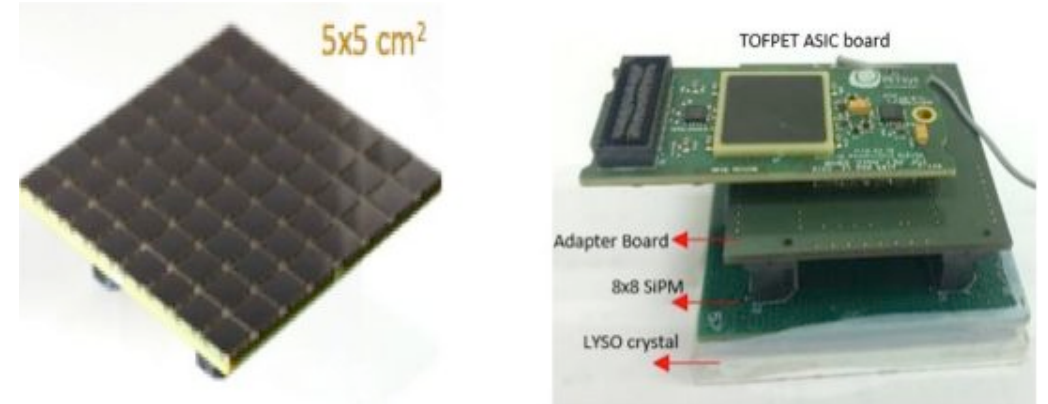

Fig. 2. Left. $8 \times 8$ SiPM array with $6.33 \mathrm{~mm}$ pitch. Right: detector block composed of LYSO crystal, $8 \times 8$ SiPMs array, an adapter board and the FEB-A ASIC board.

\section{Materials and Methods}

\subsection{ASIC readout}

Reading and digitizing the signals coming from the SiPM photosensors is carried out using the ASIC chip TOFPET1 from PETsys (Lisbon, Portugal). The input stage of the ASIC is composed of dedicated amplifiers, discriminators and Time-to-Digital Converters (TDC) [4]. The charge is provided using the Time-over-Threshold (ToT) method, which measures the time in which a signal stays above a predefined threshold. Information on all channels is managed by an intermediate board, which packs the information of all ASICs into a single data frame $\left(72 \times 10^{6}\right.$ events/s output rate) and sends it to the DAQ board $\left(250 \times 10^{6}\right.$ events/s output rate), connected to a PC via 
PCI-e link. Figure 2 shows the photograph of the SiPM array on the left, and the detector block including the ASIC and an adaptor board on the right.

Aiming to build detectors suitable for clinical applications, a large photosensor area is demanded. The photosensors used throughout all the measurements were the SensL J-series $8 \times 8$ SiPM array with $6 \times 6 \mathrm{~mm}^{2}$ active area per SiPM and pitch of $6.33 \mathrm{~mm}$. This array exhibits low dark count rate $\left(30 \mathrm{kHz} / \mathrm{mm}^{2}\right.$ at the breakdown voltage), uniform bias voltage and low temperature drift. This makes it suitable for applications with monolithic blocks in which the SNR is relative small.

\subsection{Set-Up}

Experiments where initially performed using LYSO crystal arrays of roughly $50 \mathrm{~mm} \times 50 \mathrm{~mm} \times 6 \mathrm{~mm}$ and 1.6 mm pixel size. This set-up facilitates the evaluation of the electronics performance channel by channel. By scanning several SiPM pairs from opposite photo-detectors, the system capability to determine CRT was precisely evaluated. We set a controlled temperature environment near $20^{\circ} \mathrm{C}$.

In addition to pixelated crystals, we also carried out tests using a monolithic LYSO scintillator with trapezoidal shape $(50 \mathrm{~mm} \times 50 \mathrm{~mm}$ exit face, $40 \mathrm{~mm} \times 40 \mathrm{~mm}$ entrance face, and $10 \mathrm{~mm}$ thickness $)$ with entrance and lateral surfaces black painted. We characterized the detector block in terms of energy and spatial resolution, by carrying out coincidence measurements with a reference detector based on the above crystal array $(50 \mathrm{~cm}$ of separation between detectors). Concerning the timing capabilities of the monolithic block, they were explored by using a single $6 \mathrm{~mm} \times 6 \mathrm{~mm} \times 15 \mathrm{~mm}$ LYSO pixel coupled to one SiPM as a reference detector. The distance between the two detectors was reduced to $20.5 \mathrm{~cm}$, increasing the count rate.

\section{Results}

\subsection{Crystal arrays}

The detector block under study shows the capability to resolve crystal pixels as small as $1.6 \mathrm{~mm}$, as depicted in Fig. 3. We have computed the boundary regions for each crystal pixel, so-called Voronoi diagrams [5]. The 32 
$1 \times 32$ pixels/regions are clearly distinguished, as depicted in Fig. 4. Energy resolution for single pixels was

2 measured to be nearing $20 \%$ before ToT calibration.
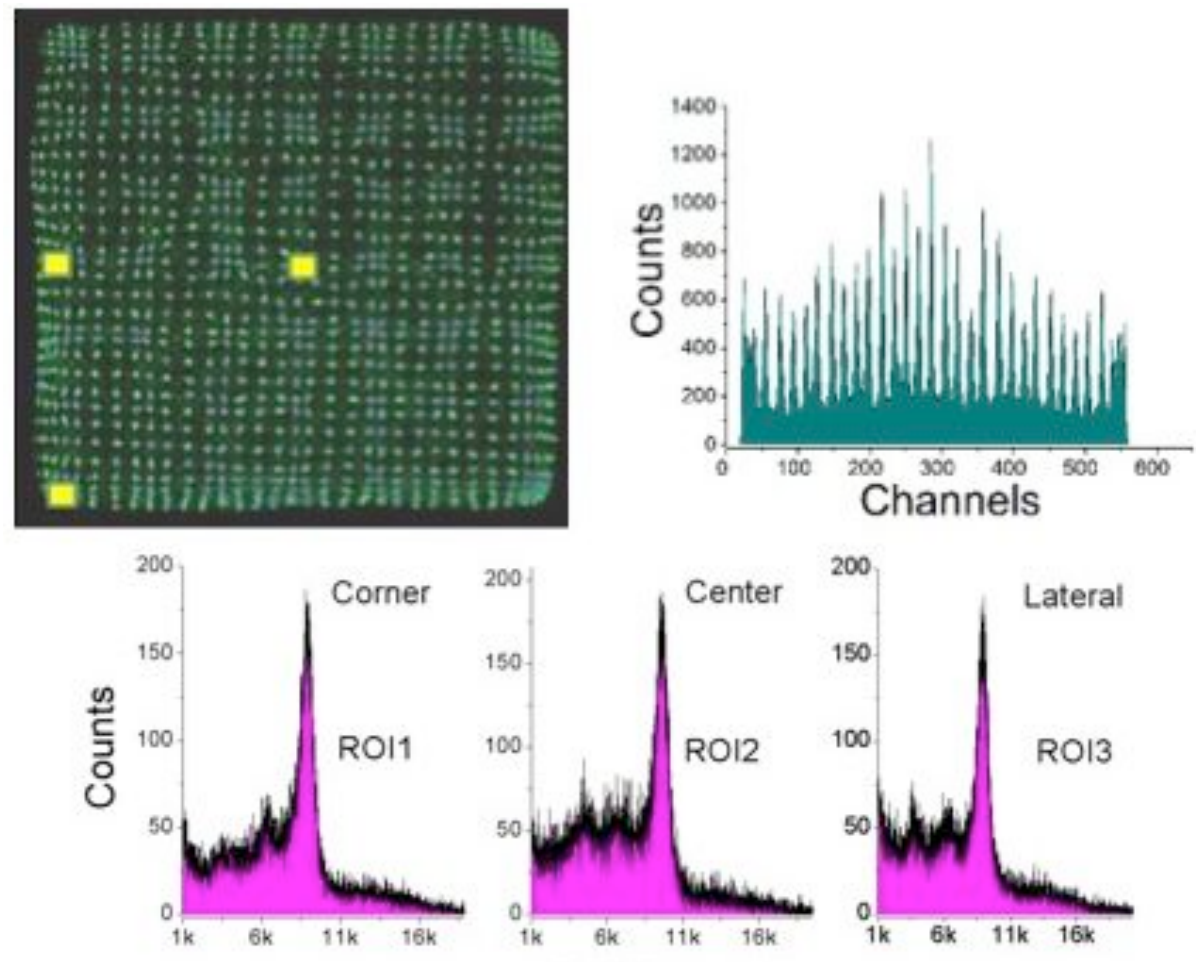

ToT channels

Fig. 3. Top-left: flood map of the $1.6 \mathrm{~mm}$ pixels for the whole detector. Top-right: a detail of an $\mathrm{X}$ projection. Bottom: energy spectra for three different small regions of interest (ROI) namely at the corner, center and lateral.

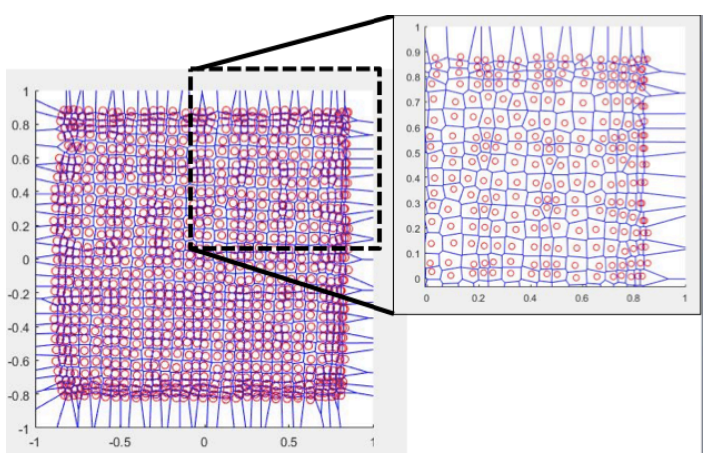

Fig. 4. Voronoi diagrams of a measurement performed with a LYSO crystal array (pixel size $1.6 \mathrm{~mm}$ ). 
For CRT measurements, a calibration of ASIC internal TDCs and Operation Amplifiers (OAs) was performed. We obtained the optimal ASIC configuration regarding CRT, by scanning the SiPM bias voltage [26-31 V], the two OA parameters named $i b l$ and $v b l$ (which are current and voltage polarization parameters, in DAC units), and the time threshold. Figure 5 shows the CRT measurements as a function of these parameters. Best CRT values were found for bias voltages nearing $29.5 \mathrm{~V}$. Optimum average $v b l$ and $i b l$ parameters were measured to be 45 and 15, respectively. Figure 5 top only shows some examples. The optimal configuration for the time threshold was determined nearing 14 DAC units above the baseline at the SiPM bias voltage of $29.5 \mathrm{~V}$. Afterwards, CRT measurements for direct channel pairs (opposite SiPMs, 64 in total) were recorded (bottomright), obtaining the best value at $330 \pm 5$ ps FWHM (mean value 553 ps, standard deviation 114 ps).
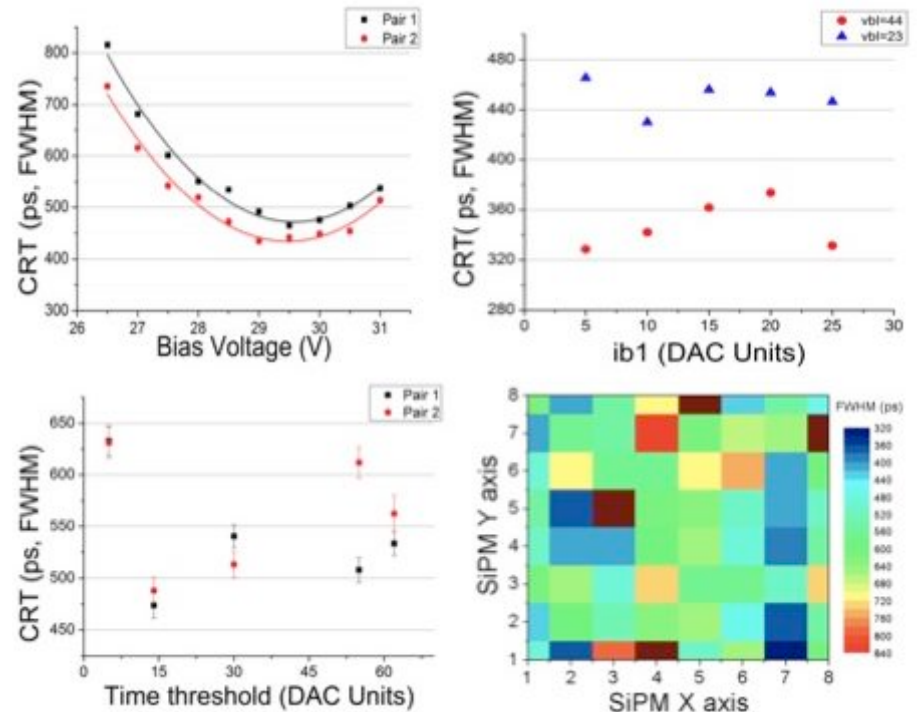

Fig. 5. Top-left: CRT as a function of SiPM bias voltage for two different SiPM pairs. Top-right: CRT as a function of current polarization parameter $i b l$ for two $v b l$ values. Bottom-left, CRT as a function of trigger threshold for two SiPM pairs. Bottom-right: CRT associated to each SiPM direct pair with the optimal OA parameters.

\subsection{Monolithic blocks}

The capabilities of the detector using a monolithic LYSO crystal were characterized by performing measurements in coincidence. $\mathrm{A}^{22} \mathrm{Na}$ array composed of $11 \times 11$ sources $(1 \mathrm{~mm}$ in diameter, $4.6 \mathrm{~mm}$ pitch, total activity near $20 \mathrm{mCi}$ ) was attached to the entrance face of the scintillation block and data were recorded for about 
15 minutes. The system spatial resolution was evaluated after considering only line of responses (LORs) with 2 angles smaller than $1.43^{\circ}$ degrees from the normal. Then by applying Center of Gravity (CoG) calculation the 3 photon impact position is determined. As depicted in the flood image in Fig. 6 top-left, it was possible to resolve 4 the $9 \times 9$ central sources. By fitting a multi-Gaussian distribution across the profile of one row of sources, an average FWHM of $2.6 \pm 0.5 \mathrm{~mm}$ was obtained. It should be noticed, that this is a preliminary estimation and 6 further improvement is expected after using more accurate mechanical alignment, other types of mechanical collimation as well as another impact algorithm estimator (see Fig. 6 bottom). Finally, an energy resolution nearing $24 \%$ was estimated.
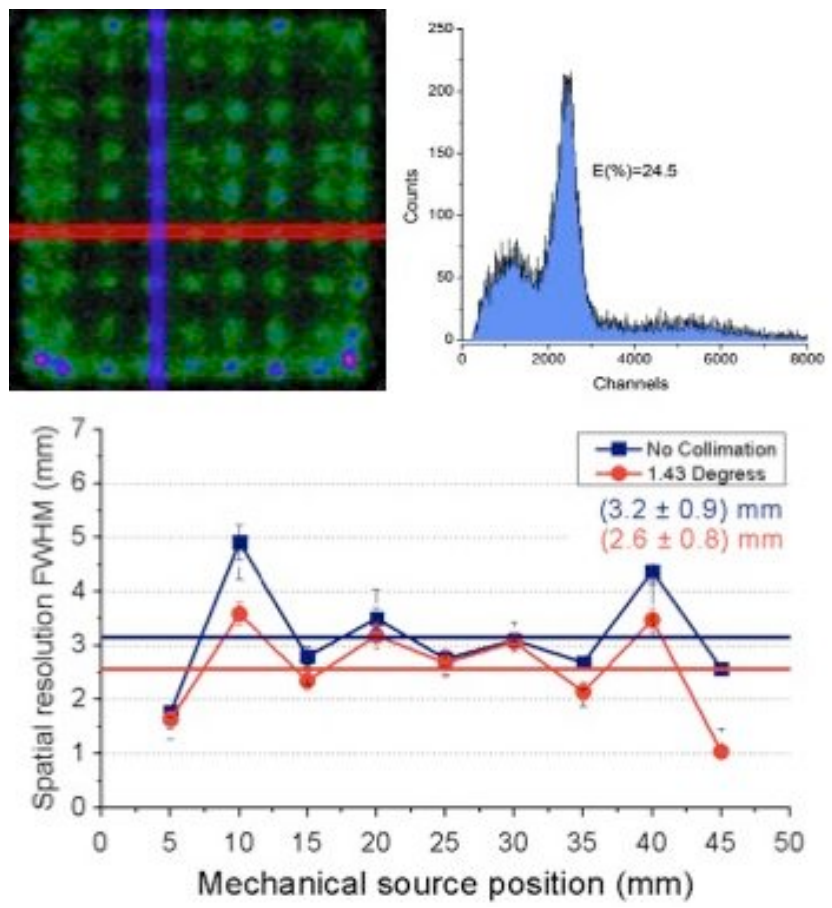

Fig. 6. Top-left: flood map for the $11 \times 11^{22} \mathrm{Na}$ sources. Top-right graph: energy profile for a small region. Bottom: mean spatial resolution measured for the horizontal sources marked on the flood plot, before and after applying electronic collimation of $1.43^{\circ}$ degrees.

The timing capabilities of the above set-up were explored after applying a calibration process to sequentially compensate both the time-walk error and the uncertainly introduced by the different time paths among SiPMs channels (time alignment) [3]. Coincidence measurements were carried out between the reference pixel detector 
1 and the monolithic block. For all the 64 channel pairs, we determined the CRT as a function of ToT values (Fig. 7 2 left). We took ToT projections of the 2D plot and calculated the centroid and FWHM using Gaussian fits. We forced time differences to be equal to zero (assume the source is on the middle of the detectors). Doing so for all 4 channels, we compensate both unwanted effects, time walk (for a single channel) and time alignment (between channels). With this information we built a table for each channel pair with the mean ToT value, the Gaussian centroid and sigma. Thus, each measurement was time walk and alignment corrected as a function of the channel pair and ToT. As plotted in Fig. 7 right, the CRT distribution after calibration is now centered at zero showing a narrower uncertainty (FWHM), except for low ToT values. We have measured a CRT value of 1.2 ns FWHM by considering only the timestamps with the highest amount of photons per impact and filtering at the $511 \mathrm{keV}$ photopeak in both coincidence detectors.
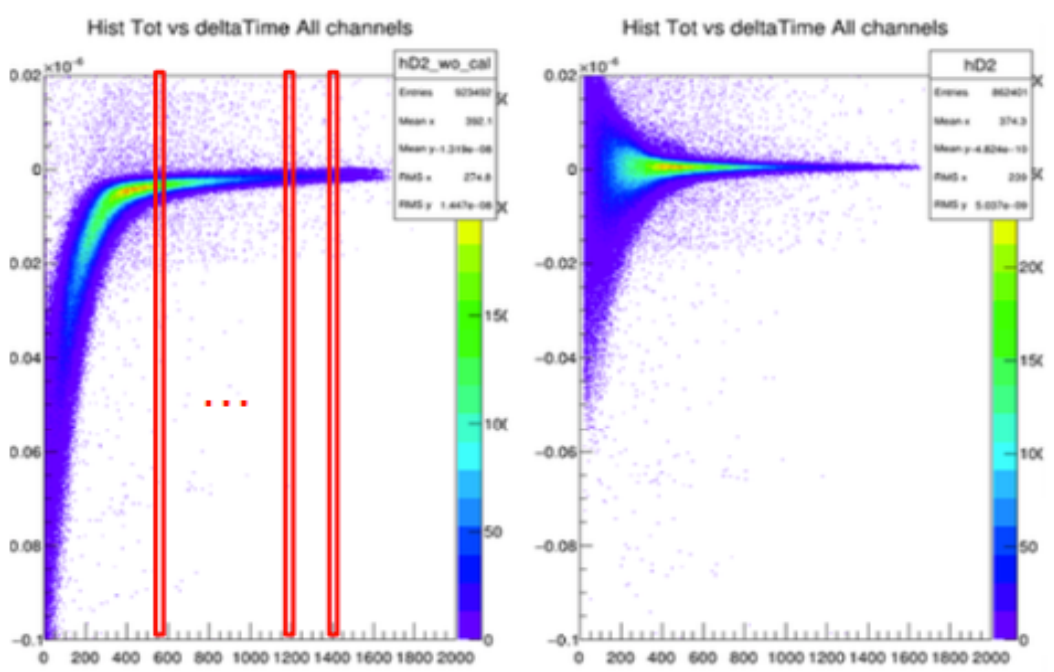

Fig. 7. Top: 2D histograms of the CRT measured as a function of the ToT collected for all channels. The left one corresponds to the one before time alignment. The right one is the result after compensating the events by the measured offset. 


\section{Conclusions}

An ASIC readout was widely evaluated during this study for its potential use in combination with monolithic scintillator crystals. Experimental results with pixelated crystals proved the ASIC capability to provide accurate results in term of energy, spatial and timing resolution. Moreover, it should be noticed the capability of the detector block to resolve $32 \times 32$ pixels, $1.6 \mathrm{~mm}$ size, using $6.33 \mathrm{~mm}$ pitch SiPMs. We did not observe significant temperature or SiPM bias dependence on the crystal pixel identification. We would also like to pinpoint the capability to reach 330 ps FWHM with the described ASIC version but using $6 \times 6 \mathrm{~mm}^{2}$ SiPM.

Concerning the measurements with monolithic blocks, promising results were obtained despite the challenges shown up when analog SiPMs and monolithic blocks are combined. The obtained result is expected to be improved after applying averaging methods to the timestamp determination instead of considering only the SiPM channel with the highest amount of light. During those measurements with monolithic blocks, we observed an average of $10 \mathrm{SiPM}$ pixel fired per impact. Early results have been shown that after applying averaging timestamp methods (energy, distance to impact, time walk compensation, etc) it is feasible to achieve accurate timing resolution [6].

Future work includes the upgrade of this current ASIC chip with the TOFPET2 ASIC that is capable of providing charge integration of signal pulses to overcome ToT limitations. This upgrade is expected to significantly improve the results, especially in terms of energy resolution. Furthermore, in the section of timing resolution, huge improvement is expected after performing threshold scans as well as after applying the previous referred averaging methods for timestamps belonging to same impact.

\section{Acknowledgments}

This project has received funding from the European Research Council (ERC) under the European Union's Horizon 2020 research and innovation program (grant agreement No 695536). It has also been supported by the Spanish Ministerio de Economía, Industria y Competitividad under Grants No. FIS2014-62341-EXP and TEC2016-79884-C2-1-R.

\section{References}


[1] S. Surti and J.S. Karp, Design considerations for a limited-angle, dedicated breast, TOF PET scanner, Phys Med Biol. 2008 Jun; 53(11): 2911-2921.

[2] S. Surti and J.S. Karp, Advances in time-of-flight PET, Phys Med, 2016 Jan; 32(1): 12-22.

[3] A. Aguilar, et al., Pilot tests of a PET detector using the TOF-PET ASIC based on monolithic crystals and SiPMs, J. Instrum. C12033, 2016

[4] T. Niknejad, et al., TOFPET2: a high-performance ASIC for time and amplitude measurements of SiPM signals in time-of-flight applications Journal of Inst., Vol. 11, (2016).

[5] K.E. Hoff, J. Keyser, M. Lin, D. Manocha, and T. Culver, Fast computation of generalized voronoi diagrams using graphics hardware, In Proceedings of the 26th annual conference on Computer graphics and interactive techniques, page 277286. 1999.

[6] H.T. van Dam, et al., Sub-200 ps CRT in monolithic scintillator PET detectors using digital SiPM arrays and maximum likelihood interaction time estimation, Phys. in Med. and Bio., 58(10), 2013 Apr; $3243-3257$. 